\title{
Radiation-induced Mutation in Man
}

MUCH effort, ingenuity and paper, in addition to flies, mice and mammalian cells in culture, have been expended in attempting to answer the important practical question of how best can the genetic risks to man of exposure to ionising radiations be estimated. Human genetic disease is a consequence of subtle changes at the gene level, or of alterations in chromosome structure or number which are detectable by the cytogeneticist. These kinds of alterations can be induced in human somatic cells exposed to radiations and there is much cytogenetic information on the radiation response of human chromosomes in blood cells, from irradiated patients or cultures, and a good start has been made on measuring radiationinduced mutation frequencies at a number of loci in rodent and human cultured cell lines. Although genetic damage in somatic cells is important to the individual, insofar as future populations are concerned it is of less consequence than damage transmitted to offspring in the germ cells. There is, however, an almost complete absence of information on radiation-induced mutation in the germ cells of man, so that in order to quantify risk in terms of mutation rate per locus per rad, it is necessary to extrapolate from experimental results obtained with other organisms.

In 1956, government committees in the United States (the BEAR Committee) and the United Kingdom (the Medical Research Council committee) relied largely on extrapolating from data obtained from irradiated fruit flies and the then emerging data from the so-called megamouse experiments undertaken at Harwell and Oak Ridge. Later government appraisals, and including those by the United Nations Scientific Committee on the Effects of Atomic Radiations (UNSCEAR), have drawn heavily on the wealth of data that has been accumulating on the radiation response of mouse genes and chromosomes. It may seem a big jump to extrapolate from observed radiation-induced mutation rates in mouse germ cells to predicted mutation rates in human germ cells, but this is the nearest comparison that can be made.

Confidence in the mouse-man extrapolation would be bolstered if it could be shown that a close similarity existed between the radiation-induced somatic genetic damage in these species. Although there is a plethora of cytogenetic information on radiation-induced chromosome damage in human blood leukocytes, it is only recently that attempts have been made to obtain equivalent information on mouse cells. This type of information helps to form a 'quantitative bridge' between mouse and man and recent data of this type would suggest that man may be equally as sensitive as the mouse to the production of specificlocus mutations which are the result of minute chromosome deletions (Brewen et al., Mutation Res., 17, 245; 1973). A somewhat different approach to the problem of extrapolation is described on page 460 of this issue of Nature, where Abrahamson, Bender, Conger and Wolff report on a remarkable relationship between mutation rates and DNA content in a wide variety of organisms, and suggest that these data may be used with confidence to extrapolate from mutation rates obtained in experimental organisms to man.
Within a species, different genes may show a wide difference in both their spontaneous mutation frequency and in the frequency with which they will mutate after exposure to radiation. For example, in the case of a number of well-studied loci in the mouse, there may be a thirty or forty-fold difference in the rate of radiationinduced mutability in different genes. Mutability is also influenced by a variety of factors, including, for example, intensity of radiation, cell stage treated, but these differences are relatively minor perturbations when considered in relation to the 1,000 -fold difference that is observed in the response between different species.

What Abrahamson et al. have now discovered is that, when forward mutation rates per locus per rad are normalised to the amount of DNA per cell nucleus, then the 1,000-fold difference between species virtually disappears, and the mutation rates in bacteria, fungi, insects, higher plants and mammals are all essentially the same. Thus, radiation-induced mutation rates per locus are directly and simply related to the DNA content of the genomea surprising finding. This remarkable association between DNA content and mutation rate now allows a further bridge to be utilised in extrapolating data from radiationinduced mutation rates in lower organisms to man. The strength of this bridge is already indicated by the fact that the mutation rates for man which may be predicted from the graph presented by Abrahamson et al. accord fairly closely with the induced mutation rate already obtained in mammalian (including man) somatic cells in tissue culture.

Why the induced mutation rate per locus should be dependent on the total DNA content of the nucleus presents something of a puzzle. Target theory, with DNA as the target, would suggest that if structural genes are the same size in bacteria as in man, then the mutation rate per locus would be independent of total DNA and equivalent between diverse species for genes coding for similar proteins. The authors point out that since this is not the case then either the nucleus, and not the locus, determines target size, or the size of the locus is proportional to total genome size for the species. This last proposal is compatible with previously proffered models in which the functional genetic unit in eukaryotes may contain not only the structural gene but also the controlling elements, thus increasing the size of the mutational unit.

H. J. E.

\section{No Trip-up This Time}

THAT transcription starts at the operator region-for the lac operator, it is a small segment of double-stranded DNA, twenty-seven bases long-is no longer in doubt (see Nature, 245, 183; 1973). When an active repressor protein is bound to the operator DNA, no transcription occurs since the promoter region to which RNA polymerase binds is generally located distal to the first (or only structural gene, so a blockade is created for any movement of RNA polymerase. When the repressor is removed, the RNA polymerase can then pass over the operator region and begin transcription. The lactose 